quence of polynomials whose roots lie on the axis of pure imaginaries and which converges uniformly in every finite region.

Hunter College

\title{
GENERALIZED LAPLACE INTEGRALS
}

R. P. BOAS, JR.

We consider the linear space $\mathfrak{S}(c)$ whose elements are functions $f(z)$ $[z=x+i y]$ which are analytic for $x>c$ and satisfy

$$
\int_{-\infty}^{\infty}|f(x+i y)|^{2} d y \leqq M, \quad x>c,
$$

where the finite number $M$ depends on the function in question. It is well known that an element $f(z)$ of $\mathfrak{S}(c)$ has boundary values $f(c+i y)$ almost everywhere on $x=c$, and that $\mathfrak{S}(c)$ is a Hilbert space if the norm of $f(z)$ is defined by

$$
\|f(z)\|^{2}=\int_{-\infty}^{\infty}|f(c+i y)|^{2} d y .
$$

Furthermore, it is known $[5$, p. 8] that if $f(z) \in \mathfrak{S}(c)$, then $f(z)$ is representable as a Laplace integral for $x>c$, in the sense that there is a unique function ${ }^{1} \phi(t)$ with $e^{-c t} \phi(t) \in L^{2}(0, \infty)$ such that

$$
\lim _{T \rightarrow \infty}\left\|f(z)-\int_{0}^{T} e^{-z t} \phi(t) d t\right\|=0 ;
$$

we shall express (2) by writing

$$
f(z)=\int_{0}^{\infty} e^{-z t} \phi(t) d t, \quad x>c .
$$

It is easily verified that the integral in (3) converges in the ordinary sense for $x>c$. A Laplace integral may be regarded as a generalized power series; the object of this note is to generalize the integral representation (3) by replacing $e^{-z t}$ by a kernel $g(z, t)$ which is in some sense "nearly" $e^{-z t}$, just as power series $\sum a_{n} z^{n}$ have been generalized ${ }^{2}$ by replacing the functions $z^{n}$ by functions $g_{n}(z)$.

Presented to the Society, September 5, 1941; received by the editors May 24, 1941.

${ }^{1}$ Unique, that is, up to sets of measure zero.

${ }^{2}$ For a bibliography of this problem, see [1]. 
We suppose that $g(z, t)$ is, for each fixed $t$ in $0<t<\infty$, an analytic function of $z$ in $x>c$; and that for each fixed $z$ in $x>c, g(z, t) \in L^{2}(0, R)$ for every $R>0$.

THEOREM 1. If for each positive $T$

$$
e^{-c t} \phi(t) \in L^{2}(0, T) \text { implies } \int_{0}^{T} g(z, t) \phi(t) d t \in \mathfrak{S}(c) ;
$$

and if there is a number $\lambda, 0<\lambda<1$, such that

$$
\left\|\int_{R}^{S} a(t)\left[e^{-z t}-g(z, t)\right] d t\right\| \leqq \lambda\left\|\int_{R}^{S} a(t) e^{-z t} d t\right\|
$$

for all $S>R>0$ and all functions $a(t) \in L^{2}(R, S)$, then there exists for each $f(z) \in \mathfrak{S}(c)$ a unique $\psi(t)$ with $e^{-c t} \psi(t) \in L^{2}(0, \infty)$ such that

$$
f(z)=\int_{0}^{\infty} g(z, t) \psi(t) d t, \quad x>c,
$$

where the integral is a mean-square limit for $x \geqq c$, and also converges in the ordinary sense for $x>c$.

In Theorems 2, 3 and 5 we shall replace the conditions of Theorem 1 by more convenient conditions; in Theorem 6 the theory will be applied to the generalized Laplace integrals recently discussed by Meijer [4] and Greenwood [7], namely

$$
f(z)=\left(\frac{2}{\pi}\right)^{1 / 2} \int_{0}^{\infty} K_{\nu}(z t)(z t)^{1 / 2} \phi(t) d t,
$$

where $K_{\nu}(z)$ is the usual notation for a Bessel function of imaginary argument [6, p. 78], and $-\frac{1}{2}<\Re(\nu)<\frac{1}{2}$ (if $\nu= \pm \frac{1}{2}$, we have (3) again).

Theorem 1 is easily proved by the method of successive approximations used for a similar purpose by Paley and Wiener [5, p. 100].

Let $f(z) \in \mathfrak{S}(c)$. Then there is a function $\phi(t)$ with $e^{-c t} \phi(t) \in L^{2}(0, \infty)$ such that

$$
f(z)=\int_{0}^{\infty} e^{-z t} \phi(t) d t, \quad \quad x>c ;
$$

here, and throughout the proof, integrals over $(0, \infty)$ are taken as mean-square limits, as in (2). Then the integral

exists; for, by (5),

$$
\int_{0}^{\infty} g(z, t) \phi(t) d t, \quad x>c,
$$




$$
\left\|\int_{R}^{S} \phi(t)\left[e^{-z t}-g(z, t)\right] d t\right\| \leqq \lambda\left\|\int_{R}^{S} \phi(t) e^{-z t} d t\right\| \rightarrow 0
$$

as $R$ and $S \rightarrow \infty$.

We now define inductively sequences $\left\{\phi_{n}(t)\right\}$ and $\left\{f_{n}(z)\right\}$ by setting $\phi_{0}(t)=\phi(t)$;

$$
f_{1}(z)=\int_{0}^{\infty} \phi(t)\left[e^{-z t}-g(z, t)\right] d t=\int_{0}^{\infty} \phi_{1}(t) e^{-z t} d t ;
$$

and generally

$$
\begin{aligned}
f_{n}(z) & =\int_{0}^{\infty} \phi_{n}(t) e^{-z t} d t, \\
f_{n+1}(z) & =\int_{0}^{\infty} \phi_{n}(t)\left[e^{-z t}-g(z, t)\right] d t .
\end{aligned}
$$

Thus

and by (5)

$$
f(z)-f_{n+1}(z)=\int_{0}^{\infty} \sum_{k=0}^{n} \phi_{k}(t) g(z, t) d t
$$

$$
\begin{aligned}
\left\|f_{n+1}\right\| & \leqq \lambda\left\|\int_{0}^{\infty} \phi_{n}(t) e^{-z t} d t\right\|=\lambda\left\|f_{n}\right\| \\
& \leqq \cdots \leqq \lambda^{n+1}\|f\| \rightarrow 0, \quad n \rightarrow \infty
\end{aligned}
$$

Consequently

$$
\left\|f(z)-\int_{0}^{\infty} \sum_{k=0}^{n} \phi_{k}(t) g(z, t) d t\right\| \rightarrow 0, \quad n \rightarrow \infty .
$$

Now

$$
\left\|\int_{0}^{\infty} \phi_{n}(t) e^{-z t} d t\right\|=\left\|f_{n}\right\| \leqq \lambda_{+}^{n+1}\|f\|
$$

and hence

$$
\left\|\int_{0}^{\infty} \sum_{m}^{n} \phi_{k}(t) e^{-z t} d t\right\| \leqq \sum_{m}^{n} \lambda^{k+1}\|f\| \rightarrow 0, \quad m, n \rightarrow \infty .
$$

Therefore, since $\mathfrak{S}(c)$ is complete, there exists $F(z) \in \mathfrak{S}(c)$ such that

$$
\left\|F(z)-\int_{0}^{\infty} \sum_{k=0}^{n} \phi_{k}(t) e^{-z t} d t\right\| \rightarrow 0, \quad n \rightarrow \infty,
$$

and

$$
F(z)=\int_{0}^{\infty} \psi(t) e^{-z t} d t, \quad e^{-c t} \psi(t) \in L^{2}(0, \infty)
$$


If we set

$$
\psi_{n}(t)=\psi(t)-\sum_{k=0}^{n} \phi_{k}(t)
$$

we then have

then by (5)

$$
\left\|\int_{0}^{\infty} \psi_{n}(t) e^{-z t} d t\right\| \rightarrow 0
$$

$$
\left\|\int_{0}^{\infty} g(z, t) \psi_{n}(t) d t\right\| \leqq(1+\lambda)\left\|\int_{0}^{\infty} e^{-z t} \psi_{n}(t) d t\right\| \rightarrow 0 .
$$

By (10) and (8) we thus have

$$
f(z)=\int_{0}^{\infty} \psi(t) g(z, t) d t,
$$

and the existence of the representation (6) is established. That (6) converges in the ordinary sense for $x>c$ follows easily from the fact that functions of $\mathfrak{S}(c)$ are represented by their Cauchy integrals [2, p. 338].

To show that the representation is unique, we have only to show that

$$
\int_{0}^{\infty} \omega(t) g(z, t) d t \equiv 0, \quad x>c,
$$

implies $\omega(t)=0$ almost everywhere. Now if (11) is true, for every positive $T$ we have, by (5),

$$
\begin{aligned}
\left\|\int_{0}^{T} \omega(t) e^{-z t} d t\right\| & \leqq\left\|\int_{0}^{T} \omega(t)\left[e^{-z t}-g(z, t)\right] d t\right\|+\left\|\int_{0}^{T} \omega(t) g(z, t) d t\right\| \\
& \leqq \lambda\left\|\int_{0}^{T} \omega(t) e^{-z t} d t\right\|+\left\|\int_{0}^{T} \omega(t) g(z, t) d t\right\| .
\end{aligned}
$$

The limit of the last term on the right is zero, and since $\lambda<1$ it follows that

$$
\int_{0}^{\infty} \omega(t) e^{-z t} d t=0, \quad x>c .
$$

Since the Laplace representation (3) is unique, $\omega(t)=0$ almost everywhere.

Theorem 2. If for $x>c$

$$
g(z, t)=e^{-z t}\{1+h(z, t)\}
$$


with

and

$$
\begin{array}{rlrl}
h(z, t) & =\int_{0}^{\infty} e^{-z u} \omega(t, u) d u, & & x>c \\
|\omega(t, u)| & \leqq \omega(u), & \\
h(z) & =\int_{0}^{\infty} e^{-z u} \omega(u) d u, & x>c,
\end{array}
$$

$$
h(c)<1,
$$

then the function $g(z, t)$ has properties (4) and (5), and consequently the representation (6) is possible and unique.

If $a(t) \in L^{2}(R, S)$, we set $a^{*}(t)=a(t)$ in $(R, S)$ and $a^{*}(t)=0$ outside $(R, S)$, and use the Parseval theorem for Laplace transforms; with $z=c+i y$,

$$
\begin{aligned}
\int_{-\infty}^{\infty} \mid \int_{R}^{S} e^{-z t} a(t) & \left.h(z, t)\right|^{2} d y \\
& =\int_{-\infty}^{\infty}\left|\int_{0}^{\infty} e^{-z t} a^{*}(t) d t \int_{0}^{\infty} e^{-z u} \omega(t, u) d u\right|^{2} d y \\
& =\int_{-\infty}^{\infty}\left|\int_{0}^{\infty} e^{-z v} d v \int_{0}^{v} a^{*}(t) \omega(t, v-t) d t\right|^{2} d y \\
& =\frac{1}{2 \pi} \int_{0}^{\infty}\left|e^{-c v} \int_{0}^{v} a^{*}(t) \omega(t, v-t) d t\right|^{2} d v \\
& \leqq \frac{1}{2 \pi} \int_{0}^{\infty}\left|e^{-c v} \int_{0}^{v}\right| a^{*}(t)|\omega(v-t) d t|^{2} d v \\
& =\int_{-\infty}^{\infty}\left|\int_{0}^{\infty} e^{-z v} d v \int_{0}^{v}\right| a^{*}(t)|\omega(v-t) d t|^{2} d y \\
& =\int_{-\infty}^{\infty}\left|\int_{0}^{\infty} e^{-z t}\right| a^{*}(t)\left|d t \int_{0}^{\infty} e^{-z u} \omega(u) d u\right|^{2} d y \\
& =\int_{-\infty}^{\infty}\left|h(z) \int_{0}^{\infty} e^{-z t}\right| a^{*}(t)|d t|^{2} d y \\
& \leqq[h(c)]^{2} \int_{-\infty}^{\infty}\left|\int_{0}^{\infty} e^{-z t}\right| a^{*}(t)|d t|^{2} d y \\
& \leqq \int_{-\infty}^{\infty}\left|\int_{R}^{S} e^{-z t} a(t) d t\right| 2 d y .
\end{aligned}
$$


Since $h(c)<1$, this gives us (5). But it also implies

$$
\begin{aligned}
\left\{\int_{-\infty}^{\infty}\left|\int_{0}^{T} \phi(t) g(z, t) d t\right|^{2} d y\right\}^{1 / 2} & \\
& \leqq\{1+h(c)\}\left\{\int_{-\infty}^{\infty}\left|\int_{0}^{T} \phi(t) e^{-z t} d t\right|^{2} d y\right\}^{1 / 2} \\
& =\frac{1}{2 \pi}\{1+h(c)\}\left\{\int_{0}^{T}|\phi(t)|^{2} e^{-2 c t} d t\right\}^{1 / 2}
\end{aligned}
$$

and hence (4).

For example, (13) is satisfied, with $c=1$, if $h(z, t)=z /\left(z^{2}+t^{2}\right)$ or $1 /(z+t)$.

TheOREM 3. If $h(z, t)$ is defined by (12) and satisfies

$$
|h(z, t)| \leqq|k(z)| l(t), \quad x>c,
$$

with $\|k(z)\|\left(\int_{0}^{\infty}|l(t)|^{2} d t\right)^{1 / 2}=\mu<(2 \pi)^{-1 / 2}$, then $g(z, t)$ has properties (4) and (5) and the representation (6) is possible.

In fact, we have

$$
\begin{aligned}
\left|\int_{R}^{S} a(t) e^{-z t} h(z, t) d t\right| & \leqq|k(z)| \int_{R}^{S} e^{-c t} l(t)|a(t)| d t \\
\left\|\int_{R}^{S} a(t) e^{-z t} h(z, t) d t\right\| & \leqq\|k(z)\|\left\{\int_{R}^{S}|l(t)|^{2} d t\right\}^{1 / 2}\left\{\int_{R}^{S} e^{-2 c t}|a(t)|^{2} d t\right\}^{1 / 2} \\
& \leqq(2 \pi)^{1 / 2} \mu\left\|\int_{R}^{S} a(t) e^{-z t} d t\right\| .
\end{aligned}
$$

This verifies (5); and (4) follows because

$$
\begin{aligned}
\left\|\int_{0}^{T} a(t) g(z, t) d t\right\| & =\left\|\int_{0}^{T} a(t) e^{-z t}[1+h(z, t)] d t\right\| \\
& \leqq\left\|\int_{0}^{T} a(t) e^{-z t} d t\right\|+\left\|\int_{0}^{T} a(t) e^{-z t} h(z, t) d t\right\| \\
& \leqq\left[1+(2 \pi)^{1 / 2} \mu\right]\left\|\int_{0}^{\infty} a(t) e^{-z t} d t\right\| .
\end{aligned}
$$


THEOREM 4. If $g(z, t)=g^{*}(z, t)$ for $t \geqq s>0$; if the representation (6) is possible and unique for $x>c^{*}$, with $g(z, t)$, for every $f(z)$ of $\mathfrak{S}\left(c^{*}\right)$; and possible and unique for $x>c$, with $g^{*}(z, t)$, for every $f(z)$ of $\mathfrak{S}(c)$ (where $\left.c<c^{*}\right)$, then the representation is also possible and unique for $x>c$, with $g(t, z)$, for every $f(z)$ of $\mathfrak{S}(c)$.

We have

$$
f(z)=\int_{0}^{\infty} g(z, t) \psi(t) d t, \quad x>c^{*}
$$

Define

$$
F(z)=f(z)-\int_{0}^{s} g(z, t) \psi(t) d t=\int_{s}^{\infty} g(z, t) \psi(t) d t=\int_{s}^{\infty} g^{*}(z, t) \psi(t) d t .
$$

The last integral defines $F(z)$ for $x>c$; and since the representation of $F(z)$ in terms of $g^{*}(z, t)$ is unique for $x>c$, the integral

$$
\int_{s}^{\infty} g(z, t) \psi(t) d t
$$

must represent $F(z)$ for $x>c$; hence (15) represents $f(z)$ for $x>c$ also.

THEOREM 5. Theorem 3 remains true if (14) is satisfied with $k(z) \in \mathfrak{S}(c)$ and $l(t) \in L^{2}(0, \infty)$.

If $k(z) \in \mathfrak{S}(c)$ we have

$$
\lim _{x \rightarrow \infty} \int_{-\infty}^{\infty}|k(x+i y)|^{2} d y=0
$$

for, if $x>c$,

$$
k(x+i y)=\int_{0}^{\infty} e^{-x t-i y t} \phi(t) d t, \quad e^{-c t} \phi(t) \in L^{2}(0, \infty) ;
$$

and so

$$
\int_{-\infty}^{\infty}|k(x+i y)|{ }^{2} d y=\frac{1}{2 \pi} \int_{0}^{\infty} e^{-2 x t}|\phi(t)|^{2} d t \rightarrow 0, \quad x \rightarrow \infty .
$$

Choose $s$ so large that

$$
\|k(z)\|\left(\int_{s}^{\infty}|l(t)|^{2} d t\right)^{1 / 2}=\mu<(2 \pi)^{-1 / 2} .
$$

Then define $h^{*}(z, t)=h(z, t)$ for $t>s, h^{*}(z, t)=0$ for $0 \leqq t \leqq s$; and 
$g^{*}(z, t)=e^{-z t}\left[1+h^{*}(z, t)\right]$. The function $g^{*}(z, t)$ satisfies the conditions of Theorem 3 , with $l(t)$ replaced by the function $l^{*}(t)$ equal to $l(t)$ in $(s, \infty)$ and to zero in $(0, s)$. On the other hand, if $b$ is so large that

$$
\left\{\int_{-\infty}^{\infty}|k(b+i y)|^{2} d y\right\}^{1 / 2}\left\{\int_{0}^{\infty}|l(t)|^{2} d t\right\}^{1 / 2}<(2 \pi)^{-1 / 2}
$$

(such a $b$ exists because of $(16)$ ), the function $g(z, t)$ satisfies the conditions of Theorem 3 with $c$ replaced by $b$. Thus the representation (6) is possible and unique with $g(z, t)$ for $x>b$, and with $g^{*}(z, t)$ for $x>c$. Theorem 5 now follows from Theorem 4 .

Let $K_{\nu}(z)$ have its usual meaning in the theory of Bessel functions $[6$, p. 78].

Theorem $6 .^{3}$ If $-\frac{1}{2}<\Re(\nu)<\frac{1}{2}$, then for every $f(z) \in \mathfrak{S}(0)$ there is a unique $\psi(t) \in L^{2}(0, \infty)$ such that

$$
f(z)=\left(\frac{2}{\pi}\right)^{1 / 2} \int_{0}^{\infty} K_{\nu}(z t)(z t)^{1 / 2} \psi(t) d t, \quad x>0,
$$

where the integral is a mean-square limit, as in (2), for $x \geqq 0$, and converges in the ordinary sense for $x>0$.

We shall show that the function $(2 z t / \pi)^{1 / 2} K_{\nu}(z t)$ satisfies the hypotheses of Theorem 5, with $c=0$. The following inequalities for $K_{\nu}(z)$ are valid for $\Re(z)>0[6$, p. $219 ; 3$, p. 658].

$$
\begin{aligned}
& (2 z / \pi)^{1 / 2} e^{z} K_{\nu}(z)=R_{0}(z), \\
& (2 z / \pi)^{1 / 2} e^{z} K_{\nu}(z)=1+R_{1}(z) /(2 z),
\end{aligned}
$$

where

$$
\left|R_{j}(z)\right| \leqq\left|\frac{\cos \nu \pi}{\cos \Re(\nu \pi)}\right|, \quad j=0,1 .
$$

If now $h(z, t)$ is defined by

$$
(2 z t / \pi)^{1 / 2} K_{\nu}(z t)=e^{-z t}[1+h(z, t)],
$$

from (20) and (19) we have, with $A(\nu)$ depending only on $\nu$,

$$
|h(z, t)| \leqq \frac{A(\nu)}{|z t|}, \quad|h(z, t)| \leqq A(\nu), \quad x>0,0<t<\infty .
$$

${ }^{3}$ A closely related theorem is given by Meijer [4, p. 603]. Meijer also obtains an inversion formula for (18). 
Hence, with some $B(\nu)$,

$$
|h(z, t)| \leqq \frac{B(\nu)}{|1+z|(1+t)}, \quad x>0,0<t<\infty,
$$

and the conditions of Theorem 5 are satisfied.

\section{REFERENCES}

1. R. P. Boas, Jr., Expansion of analytic functions, Transactions of this Society, vol. 48 (1940), pp. 467-487.

2. E. Hille and J. D. Tamarkin, On the absolute integrability of Fourier transforms, Fundamenta Mathematicae, vol. 25 (1935), pp. 329-352.

3. C. S. Meijer, Asymptotische Entwicklungen von Besselschen, Hankelschen und verwandten Funktionen, I, II, III, IV, Proceedings of the Section of Sciences, Koninklijke Akademie van Wetenschappen te Amsterdam, vol. 35 (1932), pp. 656-667, 852-866, 948-958, 1079-1090.

4. - Ueber eine Erweiterung der Laplace-Transformation, I, II, Proceedings of the Section of Sciences, Koninklijke Akademie van Wetenschappen te Amsterdam, vol. 43 (1940), pp. 599-608, 701-711.

5. R. E. A. C. Paley and N. Wiener, Fourier Transforms in the Complex Domain, American Mathematical Society Colloquium Publications, vol. 19, 1934.

6. G. N. Watson, A Treatise on the Theory of Bessel Functions, 1922.

7. R. E. Greenwood, Hankel and other extensions of Dirichlet's series, Annals of Mathematics, (2), vol. 42 (1941), pp. 778-805.

Duke University 